\title{
On the equilibria of the MAPK cascade: cooperativity, modularity and bistability
}

\author{
C. Russo ${ }^{1,2}$, C. Giuraniuc $^{1}$, R. Blossey ${ }^{1}$ and J.-F. Bodart ${ }^{1,2}$ \\ ${ }^{1}$ Biological Nanosystems, Interdisciplinary Research Institute USR3078, \\ 50 Avenue Halley, F-59658 Villeneuve d'Ascq, France \\ ${ }^{2}$ Laboratory of Division Signal Regulation, EA 4020, Building SN-3, \\ University of Sciences and Technology Lille, 59655 Villeneuve d'Ascq, France
}

(Dated: November 14, 2018)

\begin{abstract}
In this paper we present a discussion of a phenomenological model of the MAPK cascade which was originally proposed by Angeli et al. (PNAS 101, 1822 (2004)). The model and its solution are extended in several respects: a) an analytical solution is given for the cascade equilibria, exploiting a parameter-based symmetry of the rate equations; b) we discuss the cooperativity (Hill coefficients) of the cascade and show that a feedforward loop within the cascade increases its cooperativity. The relevance of this result for the notion of modularity is discussed; c) the feedback model for cascade bistability by Angeli et al. is reconsidered. We argue that care must be taken in modeling the interactions and a biologically realistic phenomenological model cannot be too reductionist. The inclusion of a time-dependent degradation rate is needed to account for a switching of the cascade.
\end{abstract}

PACS numbers: 87.16.Xa;87.16.-b;87.17.Aa 


\section{INTRODUCTION}

The MAPK (Mitogen-Activated Protein Kinase) cascade is a paradigmatic signaling cascade which plays a crucial role in many aspects of cellular events [1]. The biological function of the cascade is to ensure that an extracellular signal arriving at the cell membrane can be transported reliably to targets in the cytoplasm and in the nucleus. The functioning of this signaling mechanism is therefore of obvious interest to biologists, since interventions in the signaling chain permit controlling physiological behaviours. From the point of view of a physicist, it is interesting to understand how different control mechanisms (nonlinearities due to cooperative molecular interactions, feedback loops etc.) shape the behaviour of the signaling chain [2, $\underline{3}, 4]$.

The MAPK cascade consists of several biochemical reaction levels in which an activated kinase at each level phosphorylates a kinase at a downstream level: the signaling chain can thus be considered as a cascade of phosphorylations. The MAPKK kinase - the activator at cascade entry - is the protein Mos in Xenopus oocytes, which underlies our work (see Figure 1). These amphibian gametes are considered as model cells, easy to process experimentally due to their size $(\sim \mathrm{mm})$, rich in the concentration of the signaling molecules (even up to $50 \mathrm{ng}$ of protein per oocyte [5]), and hence signaling events can be reliably modeled by ODE-models for the reaction kinetics.

Understanding the control mechanisms involved in the MAPK signaling cascade has been a major research topic in recent years in the emerging field of systems biology, giving evidence that several aspects of the cascades can be both studied in vivo and modeled mathematically. The key work in this respect was the paper by Huang and Ferrell [6] which developed a detailed kinetic model for the so-called ultrasensitivity of the cascade, based on experimental data obtained for the cascade in Xenopus. The notion of 'ultrasensitivity' refers to the steepness of the sigmoidal stimulus/response-curve which is the hallmark of a signaling cascade: a very nonlinear response ensures an effective response to a signal received by the cell.

In the context of the transformation of a gradual stimulus into a nonlinear response curve, the question of the control of the cascade has attracted the interest of experimenters and theorists alike. In fact, depending on how the cascade is 'implemented' within other network

elements, it can function as a switch, an amplifier or feedback-controller [7, 8, 9]. This has 
led to the view that the cascade can be considered as a 'module' inserted in a network of interactions. In particular, the role of positive and negative feedback loops in conjunction with the cascade is a topic of continuing interest [10, 11]. More recently, also the role of stochastic effects in the cascade have been considered [12], as well as the spatial progression of the signaling cascade from the plasma membrane to the nucleus [13].

In order to be able to develop increasingly quantitative comparisons between experiment and models, it is useful to understand the properties of the models as well as possible; a recent prime example in this context is the work by Ventura et al. [14]. Due to the complexity of the systems addressed, it is in fact rare that analytical calculations can be made. With this work we start a series of investigations on phenomenological models for the MAPK cascade. First, we present an exact treatment of the MAPK cascade equilibria based on a phenomenological model introduced in ref. [15].

Here we show a) that for the case of a Michaelis-Menten kinetics, the equilibria of the cascade can be computed analytically by exploiting a parameter-based symmetry of the equations. (For a simple linear kinetics, this is a text book matter, see [16].) We then further address the two following issues: b) how can the cooperativity of the cascade be changed? We show that by an indirect feedforward loop, the input signal - response curve of the cascade can become more pronounced, i.e. acquire a higher effective Hill coefficient; this results has repercussions on the notion of modularity of the cascade. Finally, c), we reconsider results by Angeli et al. on the feedback-induced bistability of the cascade and introduce a simple biological mechanism by which the cascade can switch off.

\section{A MICHAELIS-MENTEN MODEL OF THE MAPK CASCADE}

Figure 2 presents the basic scheme of the interactions in the cascade as we discuss it here. The cascade is initiated by Mos, of which the concentration $x$ is taken first as given (i.e., we neglect the kinetics of Mos for the moment). Mos activates the phosphorylations of MEK, $y_{1}$, whose once- and doubly-phosphorylated forms are denoted by $y_{2}$ and $y_{3}$, respectively. The second level of the cascade is a repetition of the first level in which now, however, the role of Mos is played by doubly-phosphorylated MEK, $y_{3}$. The second layer of the cascade is the sequence of phosphorylations of MAPK, denoted by $z_{1}$, leading to $z_{2}$ and $z_{3}$ in complete analogy to the MEK-level of the cascade (Figure 2 A). Indicated in Figure 2 B) is the case 
in which Mos can also act within the cascade by promoting phosphorylation of downstream targets in an indirect way [17]. Finally, Figure 2 C) shows that the cascade output, $z_{3}$, can act back on the input level $x$ by way of a feedback loop and hence affect the concentration of Mos - we will turn to this case in the discussion of bistability.

Following [15] we write the kinetic equations for the system as follows

$$
\begin{aligned}
& \dot{y}_{1}=\frac{V_{6} y_{2}}{K_{6}+y_{2}}-\frac{V_{3} x y_{1}}{K_{3}+y_{1}} \\
& \dot{y}_{2}=-\left(\dot{y}_{1}+\dot{y}_{3}\right) \\
& \dot{y}_{3}=\frac{V_{4} x y_{2}}{K_{4}+y_{2}}-\frac{V_{5} y_{3}}{K_{5}+y_{3}} \\
& \dot{z}_{1}=\frac{V_{10} z_{2}}{K_{10}+z_{2}}-\frac{V_{7} y_{3} z_{1}}{K_{7}+z_{1}} \\
& \dot{z}_{2}=-\left(\dot{z}_{1}+\dot{z}_{3}\right) \\
& \dot{z}_{3}=\frac{V_{8} y_{3} z_{2}}{K_{8}+z_{2}}-\frac{V_{9} z_{3}}{K_{9}+z_{3}}
\end{aligned}
$$

where the $V_{i}$ and $K_{i}$ are reaction speeds and equilibrium constants, respectively, and where the numbering of the reactions follows the scheme by [11] which is also used in ref. [15]. This scheme simply numbers the reactions sequentially layer by layer, first all phosphorylations, and then all dephosphorylations.

Eqs.(2) and (5) are a consequence of the conservation of the total number of the proteins MEK and MAPK, i.e.,

$$
\sum_{i=1,2,3} y_{i}=y_{T}, \quad \sum_{i=1,2,3} z_{i}=z_{T}
$$

Thus, $\dot{y}_{2}=-\left(\dot{y}_{1}+\dot{y}_{3}\right)$, and likewise for $\dot{z}_{2}$. The system of kinetic equations hence reduces to four independent equations only.

We now show that the equilibria of the MAPK-cascade can be determined analytically in an exact way; given that the kinetics is nonlinear, this is a non-trivial result. To demonstrate it we begin with the equation for $\dot{y}_{1}$ (i.e., non-phosphorylated MEK), eq.(1). Dividing this 
equation by $V_{6}$ and redefining the variables via

$$
x^{\prime} \equiv \frac{V_{3}}{V_{6}} x, \quad y_{i} \equiv y_{i}^{\prime} y_{T}, \quad i=1,2,3
$$

we find

$$
\frac{y_{T}}{V_{6}} \dot{y}_{1}^{\prime}=\frac{y_{2}^{\prime} y_{T}}{K_{6}+y_{2}^{\prime} y_{T}}-x^{\prime} \frac{y_{1}^{\prime} y_{T}}{K_{3}+y_{1}^{\prime} y_{T}} .
$$

The kinetic parameters and reaction speeds are as estimated in the Supplementary Material of ref. [15]. Although not exact, $K_{3}=K_{6}=y_{T}$, is a reasonable assumption which we follow throughout this paper. Making use of this symmetry, the equation then simplifies to

$$
\frac{y_{T}}{V_{6}} \dot{y}_{1}^{\prime}=\frac{y_{2}^{\prime}}{1+y_{2}^{\prime}}-x^{\prime} \frac{y_{1}^{\prime}}{1+y_{1}^{\prime}} .
$$

What remains is to redefine time according to $t^{\prime}=\left(V_{6} / y_{T}\right) t$. Dropping the primes we obtain

$$
\dot{y}_{1}=\frac{y_{2}}{1+y_{2}}-x \frac{y_{1}}{1+y_{1}} .
$$

Due to the further parameter symmetries deduced from experiment by Angeli et al., $\left(V_{3}=\right.$ $\left.V_{4}, V_{5}=V_{6}, K_{3}=K_{4}=K_{5}=K_{6}=y_{T}\right)$, the same procedure also works for the equations for $\dot{y}_{2}$ and $\dot{y}_{3}$.

In fact, the same strategy can also be applied to the equations for $z_{i}, i=1,2,3$. The only differences now are that we have already redefined the variable $y_{3}$ and time $t$; this means that the equations for $z_{i}$ do have to contain two additional parameters which reflect the different timescales of the dynamics for $y_{i}$ and $z_{i}$, and the concentration scales of the $y_{i}$ and $z_{i}$. These two new parameters are given by

$$
v \equiv \frac{V_{7}}{V_{10}} y_{T}, \quad \tau \equiv \frac{V_{6}}{V_{10}} \frac{z_{T}}{y_{T}} .
$$

For example, the equation for $z_{1}$ reads

$$
\tau \dot{z}_{1}=\frac{z_{2}}{1+z_{2}}-v y_{3} \frac{z_{1}}{1+z_{1}}
$$

and as before, due to the parameter symmetries $\left(K_{7}=K_{8}=K_{9}=K_{10}=z_{T}, V_{7}=V_{8}, V_{9}=\right.$ $\left.V_{10}\right)$, the same procedure can be applied to the equations for $z_{2}$ and $z_{3}$.

We have been able to rewrite the system of four equations (plus two which are the trivial consequence of the conservation laws) with two parameters only, whereby only one of them affects the time course of the cascade. This is clearly possible in an exact way only for the 
parameter symmetries uncovered by Angeli et al. We believe, however, that this does not constitute a major restriction for our intention to perform analytic calculations. In case the parameters do deviate from the exactly symmetrical values, our calculation can be used to develop an expansion in the perturbed parameters.

In a subsequent step we can now profit from the fact that the equations have acquired a homogeneous form in the variables

$$
w_{i} \equiv \frac{y_{i}}{1+y_{i}}, \quad q_{i} \equiv \frac{z_{i}}{1+z_{i}}
$$

Noting further that the time derivative, e.g. of $w_{i}$, is given by

$$
\dot{w}_{i}=\frac{\dot{y}_{i}}{\left(1+y_{i}\right)^{2}}
$$

and similar for the $\dot{q}_{i}$, we find that the model can be cast into the simple form

$$
\begin{aligned}
& \dot{w}_{1}=\left(1-w_{1}\right)^{2}\left(w_{2}-x w_{1}\right) \\
& \dot{w}_{2}=\left(1-w_{2}\right)^{2}\left(x w_{1}+w_{3}-x w_{2}-w_{2}\right) \\
& \dot{w}_{3}=\left(1-w_{3}\right)^{2}\left(x w_{2}-w_{3}\right) \\
& \tau \dot{q}_{1}=\left(1-q_{1}\right)^{2}\left(q_{2}-s q_{1}\right) \\
& \tau \dot{q}_{2}=\left(1-q_{2}\right)^{2}\left(s q_{1}+q_{3}-q_{2}(1+s)\right) \\
& \tau \dot{q}_{3}=\left(1-q_{3}\right)^{2}\left(s q_{2}-q_{3}\right)
\end{aligned}
$$

where

$$
s \equiv v \frac{w_{3}}{1-w_{3}}
$$

From these equations, the fixed-point conditions $\dot{w}_{i}=\dot{q}_{i}=0$ can now easily be read off. Note that since $w_{i} \leq 1 / 2$, zeroes can only appear in the right-most bracket of each equation. For the $w_{i}$ one has

$$
w_{3}=x w_{2}=x^{2} w_{1}
$$


which expresses the character of the phosphorylation mechanism in a very clear way (and in fact, analogously to the linear kinetics [16]). Similarly, for the $q_{i}$

$$
q_{3}=s q_{2}=s^{2} q_{1}
$$

In order to determine the fixed-point values of these quantities explicitly, we have to invoke the constraints on $y_{i}$ and $z_{i}$, which have to be expressed in terms of the new variables. The condition

$$
y_{1}+y_{2}+y_{3}=1
$$

becomes in terms of $w_{i}$

$$
\frac{w_{1}}{1-w_{1}}+\frac{w_{2}}{1-w_{2}}+\frac{w_{3}}{1-w_{3}}=1
$$

Putting in the fixed-point conditions for $w_{2}$ and $w_{3}$, this equation turns into a cubic equation for $w_{1}$,

$$
4 x^{3} w_{1}^{3}-3 x\left(1+x+x^{2}\right) w_{1}^{2}+2\left(1+x+x^{2}\right) w_{1}-1=0 .
$$

Due to the symmetry of the first and the second level of the cascade, the corresponding equation for $q_{1}$ is obtained by replacing $w_{1}$ by $q_{1}$, and $x$ by $s$.

Clearly, the cubic equations for $w_{1}$ and $q_{1}$ can be solved exactly with textbook formulae. There is a unique real solution which fulfills the condition $w_{1}(x=0)=1 / 2$, as follows from a study of eq.(27) near $x=0$. Since for $x \rightarrow 0, w_{1} \rightarrow 1 / 2$, we can neglect the cubic and quadratic terms and find

$$
w_{1}=\frac{1}{2} \frac{1}{1+x+x^{2}}
$$

which is a result analogous to the linear kinetics. Figure 3 (top) compares eq.(28) with the numerical solution to eq.(27). One sees that also for large values of $x$, the full solution asymptotically approaches the profile given by (28). We note that the two other solutions to eq.(27) are singular for $x \rightarrow 0$, as can be seen by neglecting the constant term -1 and solving the remaining quadratic equation. For increasing values of $x$, the solutions become complex.

From the fixed-point conditions $w_{2}=x w_{1}$ and $w_{3}=x^{2} w_{1}$ one immediately obtains from eq. (28)

$$
w_{2}=\frac{1}{2} \frac{x}{1+x+x^{2}},
$$

and

$$
w_{3}=\frac{1}{2} \frac{x^{2}}{1+x+x^{2}} .
$$


Thus we see that while $w_{1}$ diminishes as a function of $x, w_{2}$ first rises linearly, and then drops $\propto 1 / x$ for large $x$, due to the depletion for the doubly-phosphorylated form. The concentration of doubly-phosphorylated MEK, by contrast, shows exactly the expected sigmoidal Hill-type profile with a Hill coefficient of two. As far as the asymptotics of the Hill coefficients is concerned, there is no difference between the linear and the Michaelis-Menten kinetics. The precise details of the concentration profiles are, however, essential in a comparison to experiment. We illustrate this point here by comparing our approximate (linear) and the exact solution of the cubic equation for the case of Michaelis-Menten kinetics.

Figure 3 (top) illustrates the difference between the approximate solution (linear) and the exact numerically calculated solution of the cubic equation for $w_{1}(x)$. If one tries to fit the curve to a pure powerlaw $w_{1}(x) \sim x^{b}$ over a range of values $1.5 \leq x \leq 3$, which is roughly an experimentally accessible window for Mos based on the parameters used and experimental data for Mos concentrations [5, 15], the best fit is obtained for a value of $b=-1.557$. Fitting of the curve over a much wider range, one order of magnitude in $x$, reveals that the value of $b$ converges to a value of two; we find $b=-1.942$. This result of Figure 3 (bottom) shows that the attribution of the Hill coefficient (the highest nonlinearity in the concentration law) from experiment is difficult since the lower order terms, which decay less rapidly, strongly affect the result.

Due to the formal identity of the equation for $q_{1}$ with that of $w_{1}$, we immediately find the analogus results for $q_{1}, q_{2}, q_{3}$, this time with $x$ replaced by $s$. If we transform the final result for $q_{3}$ back to $z_{3}$, we find

$$
z_{3}=\frac{s^{2}}{2\left(1+s+s^{2}\right)}
$$

which, due to the quadratic dependence of $s$ from $x$ is actually a sigmoidal function with a Hill coefficient of four, as is common for the MAPK cascade; see Figure 4. Again, there is no difference in the asymptotic value of the Hill coefficient between the linear and the exact cubic case.

\section{INCREASING COOPERATIVITY, BREAKING MODULARITY}

We now turn to the second question we want to address: how can one increase the Hill coefficient, i.e. the cooperativity of the cascade? From the foregoing section we have found that the Michaelis-Menten MAPK cascade has a maximal Hill coefficient of four, the highest 
nonlinearity in the denominator of eq.(31). Evidently, the effectivity of the cascade is based on a sufficiently steep rise in concentration of MAPK at threshold. Is there a way to increase this steepness, i.e. having a still higher cooperativity?

In order to answer this question it is useful to investigate the impact of possible modifications of the cascade. Two cases may be distinguished: (i) additional levels of phosphorylation and (ii) rewirings of the cascade by the introduction of feedback and/or feedforward loops.

In the first case one can either have more phosphorylation steps within one given level, or an increase in the number of cascade levels. Although both will lead to an increase in cooperativity - increase the Hill coefficient - but this comes at a cost.

If more phosphorylation levels are introduced, the unphosphorylated and maximally phosphorylated states become disfavoured with respect to intermediate states, since the overall population of these states has thus to go down for a given constant number of proteins. More levels thus means favoring the intermediate states, while it is the entry and exit states that are really important. The function of the intermediate level is just to separate entry and exit state by separating them from each other - but the separation should not be too 'large', i.e. involve too many states.

On the other hand, an increasing the number of cascade levels requires that another molecular partner has to be involved. This would have as a consequence a decrease in the 'robustness' of the cascade. The notion of robustness is used here in the sense that building a cascade based on many different molecules increases the probability of cascade failure.

By contrast, feedback and feedforward loops are schemes through which the cascade can, in principle, increase its cooperativity without increasing the type of molecules intervening and maintaining both phosphorylation levels and cascade levels.

We now show that a feedforward loop in the cascade can indeed lead to an increase of cooperativity. Such a loop may be introduced in the cascade when one considers Mos to act through two opposite pathways within the cascade. It has been observed that the MAPKKKinase phosphatases and/or MAPK phosphatases are down-regulated and inhibited for MAPK activation, because MAPKKKinase introduced experimentally fails to properly activate MAPK on its own [17]. Similar results have been obtained in Xenopus oocytes [18]. As proposed in in mice oocytes Mos may intervene at one of the downstream levels by affecting the phosphorylation/dephosphorylation equilibrium in a concentration-dependent manner, by favouring phosphorylation such that the feedforward loop stabilizes $z_{3}$, as sketched in 
Figure 2 B).

In the context of our phenomenological model this shift of the phosphorylation equilibria leads to an effective concentration dependence of the kinetic parameters $V_{9}$ and $V_{10}$. We propose therefore the replacement of the reaction constants

$$
V_{9} \rightarrow V_{9}(x)=\frac{V_{9}^{*}}{K^{*}+x}, \quad V_{10} \rightarrow V_{10}(x)=\frac{V_{10}^{*}}{K^{*}+x}
$$

This modification of the reaction constants increases the cooperativity of the network, as characterized by the Hill coefficient, from four to six. The reason for this is the inverse polynomial dependence we assumed for simplicity. The same effect would be brought about by a similar intervention at the level of MEK.

This result has an important consequence on the idea of 'modularity' of the cascade. As argued in the introduction, the ubiquitous presence of the MAPK signaling pathway and its nonlinear characteristics make it attractive to consider it as a recurrent 'invariable module' that is programmed at cascade entry, and whose output interacts with other network elements. Our simple argument above reveals that an intervention inside the cascade, by affecting the phosphorylation/dephosphorylation equilibrium, can have a significant effect on the output (a more nonlinear signal). In this context, it would not make sense anymore to consider the MAPK cascade as an invariable modular element.

\section{BISTABILITY: RECONSIDERING THE DE ANGELI-MODEL}

The foregoing discussion has established how a gradual signal of Mos, $x$, is transformed into a response downstream of the cascade. We now discuss what happens when this nonlinear signal transformation is embedded into a circuit via a feedback loop, see Figure 2 C).

In this loop, the cascade output interacts back on the input, $x$, and therefore favors the production of $x$, autoactivating the cascade.

In order to implement the feedback loop, one has to postulate a dynamics of Mos. Angeli et al. chose the phenomenological expression [15].

$$
\dot{x}=-\gamma \frac{x}{K_{2}+x}+\tilde{V}_{0} z_{3}(x) x+\tilde{V}_{1}
$$

In this model, the first term describes the degradation of Mos with a Michaelis-Menten kinetics. The second term gives the feedback activation of Mos by MAPK, the output of the 
cascade. The function $z_{3}(x)$ can be calculated exactly for both the linear kinetics as well as for the Michaelis-Menten kinetics, as shown before. Finally, the last term in the equation describes a basal production of Mos due to translation from its mRNA. We note that the parameters in this equation have been renamed with respect to the equations before, taking into account that we had normalized the variables previously.

In a subsequent erratum published on their website, the authors modified this equation by changing the feedback term into $\tilde{V}_{0} z_{3}(x)$. We now discuss the consequences of this change. We start out from a yet slightly modified version of the Mos-dynamics, namely

$$
\dot{x}=-\gamma x+\tilde{V}_{0} z_{3}(x) x
$$

i.e., we include only a linear degradation term in $x$, and drop the constant production term $\tilde{V}_{1}$, since it has no qualitative effect on the dynamics.

Figure 5 (top) shows the two curves $\gamma x$ and $\tilde{V}_{0} z_{3}(x) x$ whose intersections determine the equilibria. It can be seen that there are at most two intersections, not three. This is due to the fact that for large $x$, since $z_{3}(x)$ saturates, both curves behave as linear functions with $z_{3}$ turning linear from below. Thus, there cannot be three solutions. This is even more apparent if one determines the mechanical potential $W$ which is easy to do now, since the dynamics in one-dimensional, i.e., we have

$$
\dot{x}=-\frac{d W}{d x} .
$$

The potential $W(x)$ is shown in Figure 5 (bottom). It shows either a single stable minimum at $x=0$ or a stable minimum at $x=0$ and an unstable maximum at a value $x=x_{u m}$.

The effect of the modification of the feedback term made by Angeli et al. now becomes clear: it is needed in order to obtain three intersections, and hence an additional, stable minimum in $W(x)$. However, the mathematical expression given by Angeli et al. still has problems. First, it is not in accord with what is shown in Fig 5 c of their paper, since there a linear curve and the curve $z_{3}(x)$ are superimposed. This would correspond to our equation (34) with the production term modified as suggested by Angeli et al. However, this modification leads to yet another problem: it means that doubly-phosphorylated MAPK can act as a source for Mos, since $\dot{x} \sim z_{3}$.

In order to be in qualitative accord with the results deduced by Angeli et al. one can 
therefore suggest a phenomenological expression

$$
\dot{x}=-\gamma x+\frac{\tilde{V}_{0} z_{3}(x) x}{\tilde{K}+x}
$$

which indeed does the give the three intersections, and hence two stable and an unstable solutions, as can be seen in Figure 6, top and bottom.

The origin of this modeling ambiguity is easy to understand. If there is only one type of Mos considered, it has to be an active form, capable of initiating the cascade. On the other hand, if it is MAPK, $z_{3}$, which activates Mos, there is evidently one species lacking, non-activated Mos. For this, one has to properly distinguish between two active forms, only one of which is stable. In order to make the Angeli et al. model more biologically realistic, therefore at least a distinction between a stable and a non-stable form of Mos is needed. Within the Angeli model, this can be implemented on the level of the degradation of Mos. We therefore close the paper by showing how the distinction between stable and non-stable Mos can lead to a switching of the cascade by acting on the degradation rate, $\gamma$.

The biological origin of this mechanism is easily justified, see the illustration in Figure 7. At early times, non-stable Mos is produced from its mRNA stock and degraded by the proteasome. Upon phosphorylation, Mos is shielded against degradation. Indeed, Mos stability is brought about by phosphorylation which leads to changes in the degradation rate of Mos [20]. Two types of kinases have been shown to be responsible for phosphorylation site modification on residues essential to Mos protein stability. These proteins are either components of the MAPK network or components of the M-phase Promoting Complex (MPF) [21, 22]. Changes in Mos phosphorylation have been observed at two key steps in the oocyte cell cycle: (1), during meiosis resumption (G2/M cell cycle transition, here denoted by $t_{1}$ ) and, (2), upon fertilization (the metaphase-anaphase transition, here denoted by $t_{2}$ ). During the time interval $t_{2}-t_{1}$, Mos is shielded against degradation, allowing its accumulation from its mRNA stock (note that this interval is on a timescale of 24 hours). Upon dephosphorylation the system goes back to a high degradation rate, driving the disappearance of Mos and the inactivation of Mos activity within 30 minutes [19, 21].

When fertilization occurs, Mos is dephosphorylated and the system goes back to an effectively high degradation rate. Note that the interval $t_{2}-t_{1}$ is on the timescale of $24 \mathrm{~h}$, while the transition from high to low degradation is much faster (30 min).

This mechanism is easily implemented in the phenomenological model. As shown in 
Figure 6 , if the degradation rate is high, the two curves $\gamma x$ and $\sim z_{3}(x) x /(\tilde{K}+x)$ do not have any common intersection other than at zero: the switch is off. Upon a decrease in the degradation rate both curves can intersect, giving rise to the three intersections discussed before. Coming back to our model, eq.(36), we recognize that it is indeed simplified in the sense that we had omitted a source for Mos, $x$, which is independent from MAPK. We now put it back in and neglect the presence of the MAPK-induced Mos production because all we need in order to realize the above scenario is a mechanism to modulate Mos concentration from low values (which corresponds to an effective high degradation rate) to high values (low rate of degradation) back to low values.

The ODE with an effective time-dependent degradation rate which contains a timevarying production and a constant degradation term has the very simple form

$$
\dot{x}=-\gamma_{e f f} x \equiv(m(t)-\gamma) x
$$

where we suppose that for all times, $m(t) \leq \gamma$. The experimental observations allow to conclude a variation of $m(t)$ such that

$$
\gamma_{e f f}(t)=\gamma_{1}, \quad t<t_{1}, \quad t>t_{2}
$$

and

$$
\gamma_{e f f}(t)=\gamma_{2}, \quad t_{1}<t<t_{2}
$$

with $\gamma_{1}>\gamma_{2}$. We thus obtain a switching-on and -off of the cascade.

Since the change in the effective degradation rate is fast, the slope of the degradation curve changes quickly from a steep to a flat profile. Therefore, the barrier separating the two stable states becomes shallow and its location moves close to the minimum of $W(x)$ at low concentration of Mos (see Figure 6). This switch enables the system to quickly exit from a now unstable state.

\section{CONCLUSION}

In this paper we have presented a discussion of a phenomenological model of the MAPK cascade. We have shown that the equilibrium states of the MAPK cascade can be determined exactly even for a model with Michaelis-Menten kinetics, a fact to our knowledge so far overlooked. 
Linear and Michaelis-Menten kinetics lead to identical asymptotic Hil coefficients. The comparison of Hill coefficients from theory to experimental data, however, must be taken with caution, since the values seen in experiment are essentially effective exponents, i.e. most likely not in the proper asymptotic regime. In this respect it is therefore very important whether a linear or Michaelis-Menten kinetics is assumed.

Further, we have shown how a simple feedforward mechanism acting on the inner levels of the cascade can increase the cooperativity of the cascade. This is an important result since it means that a 'simple' rewiring of the cascade can increase cooperativity without the addition of new cascade levels or phosphorylation steps.

This result has two important consequences. The first is obviously again for experiment, since a high measured value of the Hill coefficient might need an explanation in terms of a more detailed study of the interactions in the network; such study is currently under way [18]. The second consequence arises for the notion of modularity. In its strongest interpretation one might wish to consider the MAPK cascade as an invariable module to be implemented in a network all 'around it'. In order to optimize the response, the feedforward mechanism we propose can be advantageous, since it increases the cooperativity of the cascade, but it does so at an expense of cascade modularity. Our finding supports the idea that it is best to understand the cascade as a particular motif within a network: a motif which has no boundaries at any level to molecular interactions across the whole network.

Finally, we pointed out that the phenomenological modeling of the feedback loop which turns the MAPK cascade into a switch by selecting two out of an infinitude of possible states of the cascade needs to be done with caution. We have put forward a discussion which addresses some problems present in the model and its solution as presented by Angeli et al. The origin of this ambiguity is the fact that the model is too reduced: a proper distinction between the different states of Mos is needed. We have argued that the Angeli model needs at least a time-dependent degradation constant in order to properly account for the cascade dynamics.

Acknowledgment. CR gratefully acknowledges support through a grant from the French National Cancer Institute, INCa. 
[1] B. D. Gomperts, I. M. Kramer and P. E. R. Tatham Signal Transduction, Academic Press, San Diego (2002)

[2] J.E. FerrellNJr., Trends Biochem. Sci. 21, 460 (1996)

[3] J.E. Ferrell Jr. and W. Xiong, Chaos, 11, 227 (2001)

[4] J. J. Tyson, K. C. Chen and B.ovak, Curr. Op. Cell Biol. 15, 221

[5] N. Sagata et al, Nature 335, 519 (1988)

[6] C.-Y. F. Huang and J. E. Ferrell, Jr., PNAS 93, 10078 (1996)

[7] N. Blüthgen and H. Herzel, in 2nd Workshop on Computation of Biochemical Pathways and Genetic Networks - Berlin: Logos, 55 (2001)

[8] N. I. Markevich, J. B. Hoek and B. N. Kholodenko, Journ. Cell Biology 164, 353 (2004)

[9] J. Gunawardena, PNAS 102, 14617 (2005)

[10] W. Xiong, J. E. Ferrell, Jr., Nature 426, 460 (2003)

[11] B. N. Kholodenko, Eur. J. Biochem. 267, 1583 (2000)

[12] X. Wang, N. Hao, H. G. Dohlmann and T. C. Elston, Biophys. J. 90, 1961 (2006)

[13] N. I. Markevich, M. I. Tayganov, J. B. Hoek and B. N. Kholodenko, Mol. Sys. Biol. doi:10.1038/msb4100108 (2006)

[14] A. C. Ventura, J.-A. Sepulchre and S. D. Merajver, PLoS Comp. Biology 4, e1000041 (2008)

[15] D. Angeli, J. E. Ferrell, Jr., and E. D. Sontag, PNAS 101, 1822 (2004)

[16] U. Alon, An Introduction to Systems Biology, CRC Press (2006)

[17] M. H. Verlhac et al., EMBO J. 19, 6065 (2000)

[18] R. Beaujois and J.F. Bodart, unpublished (2009)

[19] J.-F. Bodart, S. Flament and J.-P. Vilain, Mol. Reprod. Dev. 61, 570 (2002)

[20] J. Sheng, A. Kumagai, W.G. Dunphy and A. Varshavsky, EMBO J.21, 6061 (2002)

[21] A. Castro et al., Mol. Biol. Cell. 12, 2660 (2001)

[22] W.T. Matten, T.D. Copeland, N.G. Ahn and G.F. Vande Woude, Dev. Biol. 179, 485 (1996) 


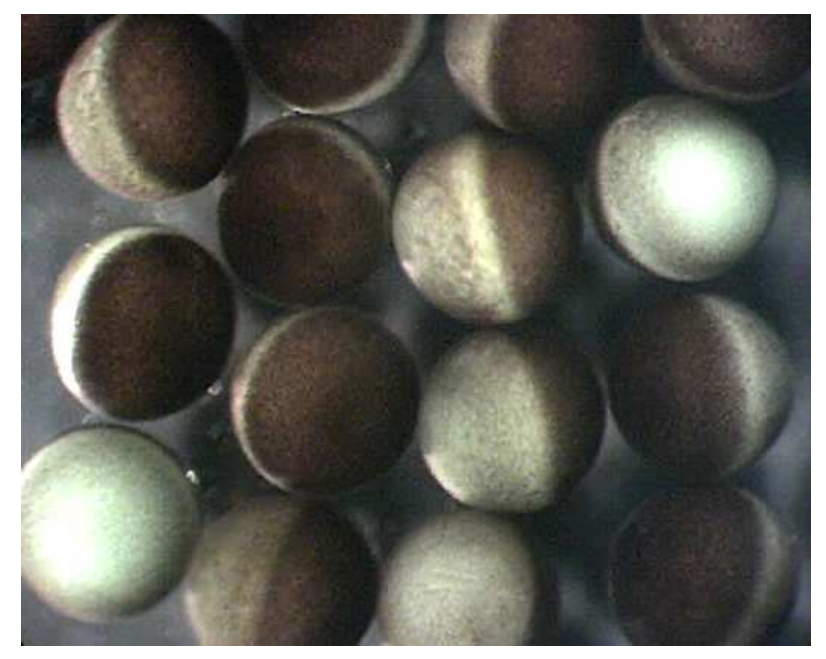

FIG. 1: Xenopus oocytes. The cells are large: a typical diameter is about $1 \mathrm{~mm}$. 
A

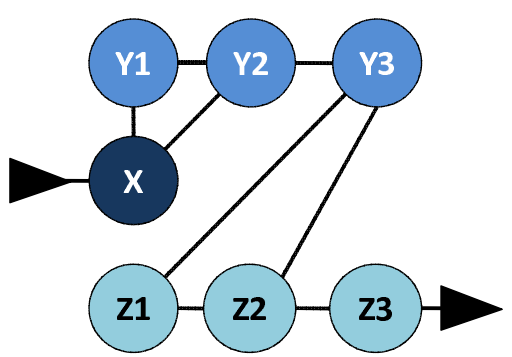

B

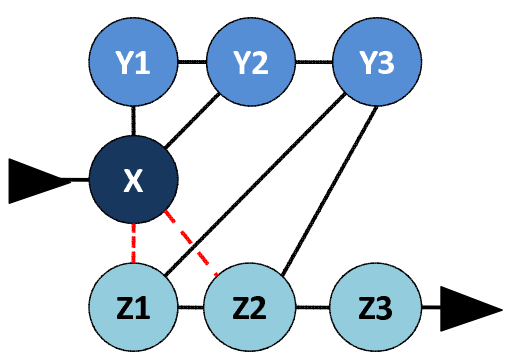

C

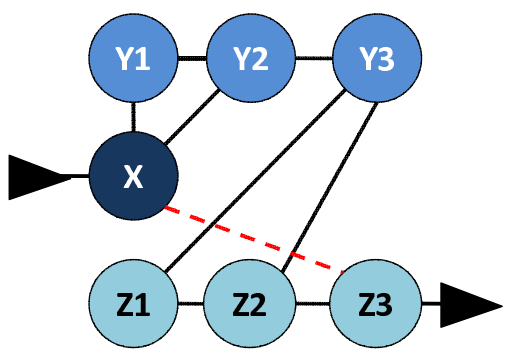

FIG. 2: (Color online.) Schematics of the MAPK cascade in the simplified model proposed in ref.[15] and extended here. The nomenclature is as follows: $x$ Mos, $y_{1,2,3}$ MEK of different, increasing phosphorylation status, and likewise $z_{1,2,3}$ for MAPK. A) shows the basic cascade: Mos promotes the phosphorylation of MEK, doubly-phosphorylated MEK, $y_{3}$, promotes the phosphorylation of MAPK. B) Mos promotes the phosphorylation of MAPK in an indirect way, as discussed in the text; C) the cascade with feedback of $z_{3}$ on $x$. 

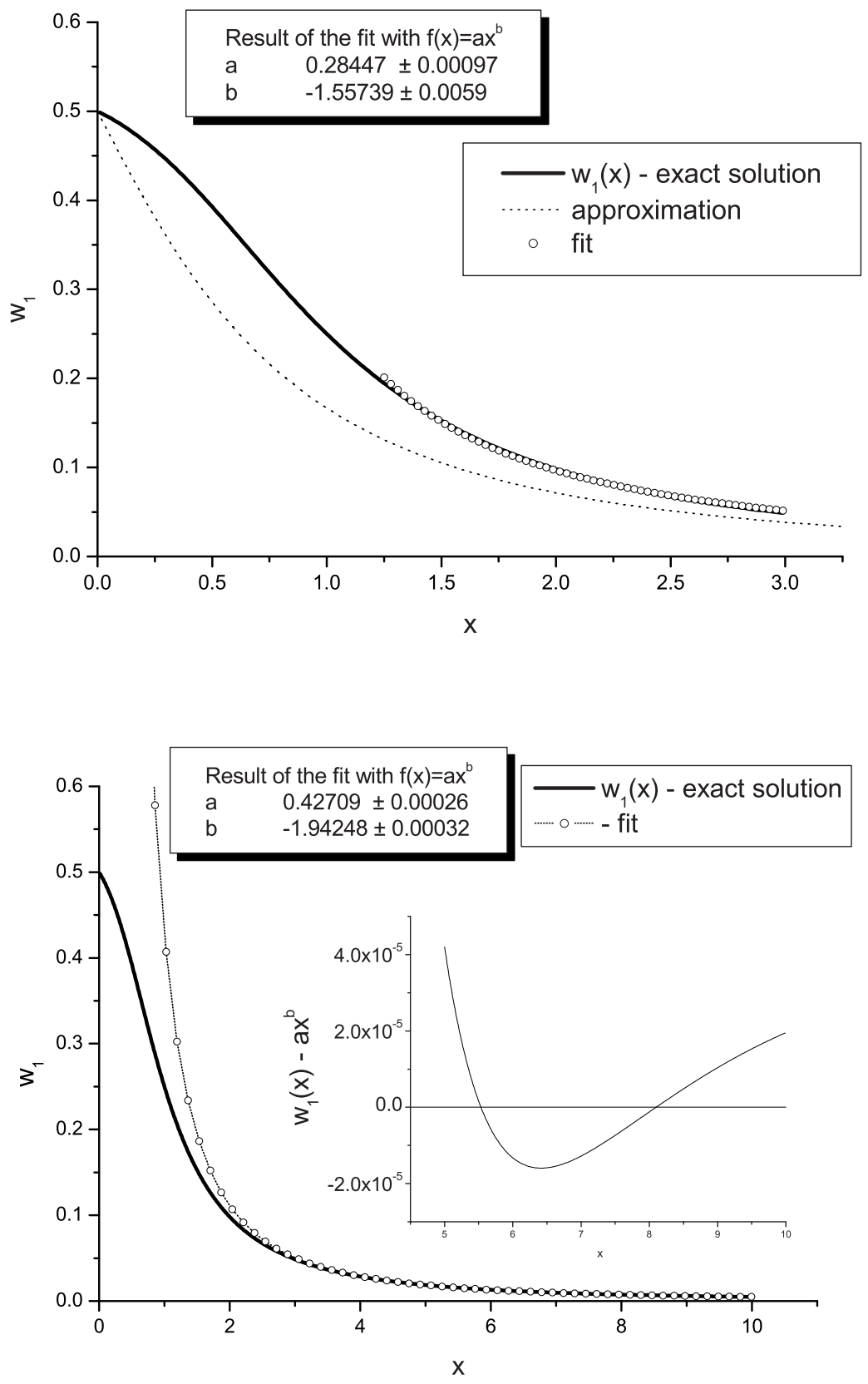

FIG. 3: The concentration of $w_{1}$ (the transformed concentration of non-phosphorylated MEK, $y_{1}$ ) as a function of concentration of Mos, $x$. The Hill exponent deviates from a value of two over the accessible concentration ranges; the asymptotic regime, for which this value would be reached, is out of range, see the discussion in the main text. 


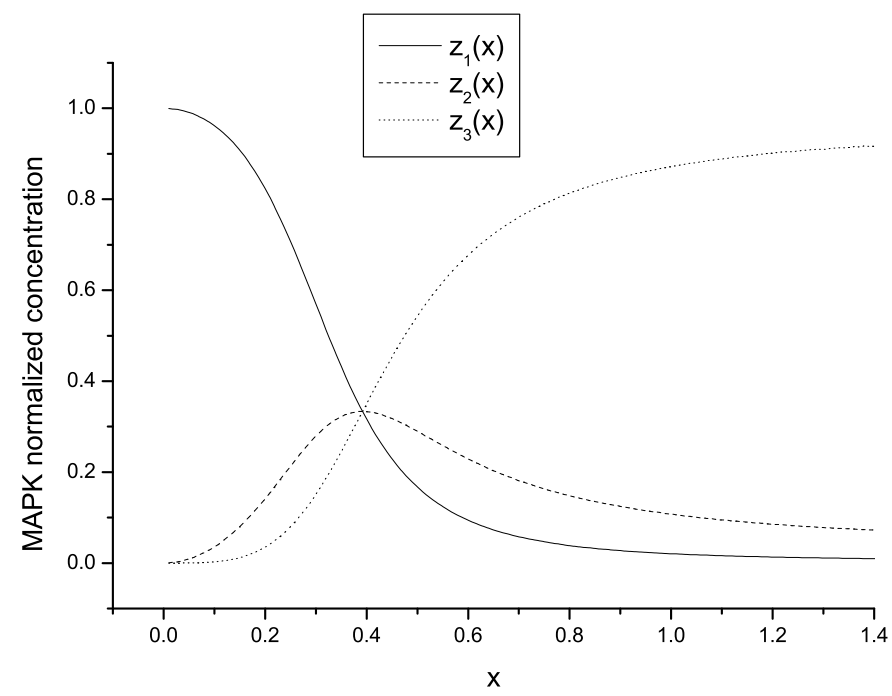

FIG. 4: The dependence of $z_{1}$ and its phosphorylated forms $z_{2}$ and $z_{3}$ as obtained from the full solution to equation (27), using the stationary state conditions, and transforming back to the original variables. 

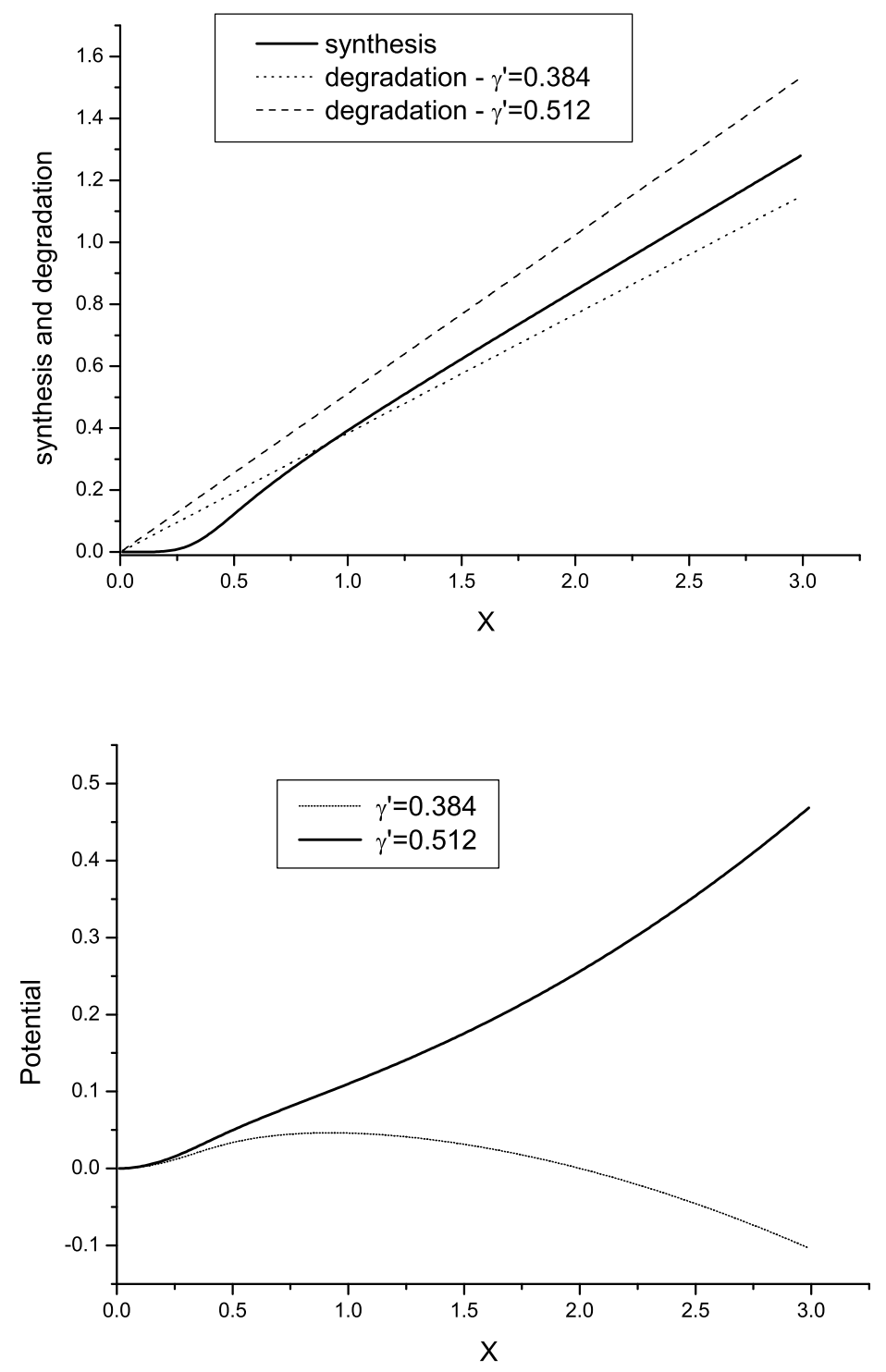

FIG. 5: Top: stationary state conditions for the model by Angeli et al. Two intersections correspond to a stable and an unstable state. See Bottom: potential. 

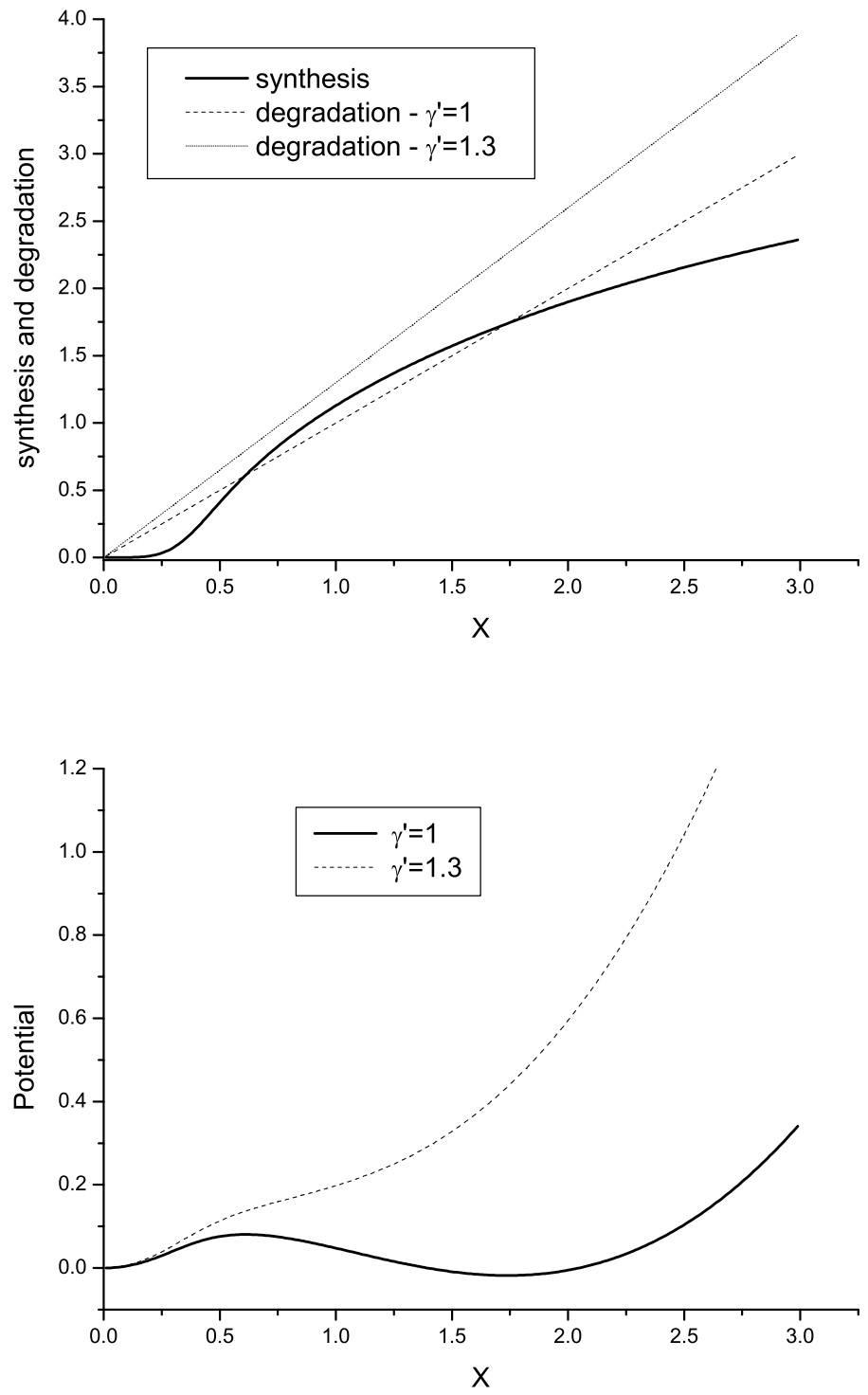

FIG. 6: Top: stationary state conditions for the model given by eq.(36). Three intersections exist. See Bottom: potential. 

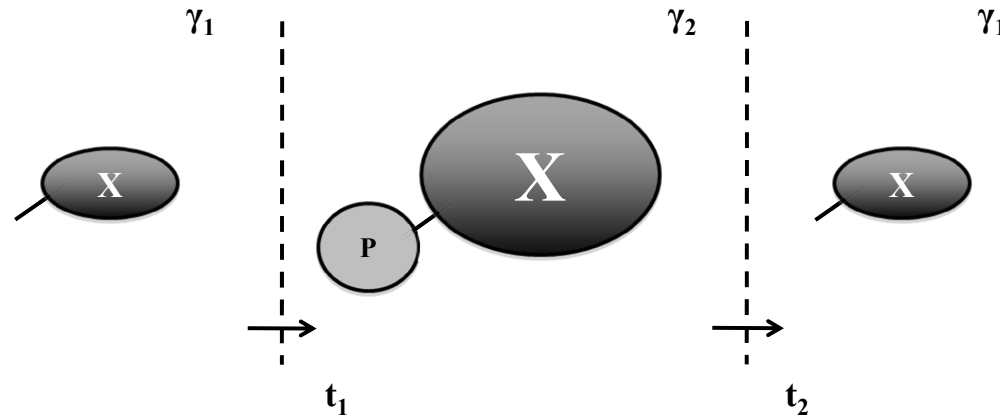

Meiotic
maturation

Fertilization

FIG. 7: The switching mechanism by modulating Mos-degradation. 